\title{
Lingual Approach to Buccally Impacted Teeth
}

\author{
${ }^{1}$ Samay Mukesh Tahilramani, ${ }^{2}$ Shashank Gaikwad, ${ }^{3}$ Grish Karandikar, ${ }^{4}$ VK Ravindranath
}

\begin{abstract}
Orthodontic management of impacted teeth is one of the most challenging aspects in terms of diagnosis and treatment planning and execution. Treatment is usually prolonged in duration and often complicated by various local factors. This article describes a simplified method toward management of an impacted tooth through the medium of a case report. A patient reported with impacted maxillary canine and an impacted mandibular premolar, was treated orthodontically. The impacted premolar was aligned using a new approach through the modification of the lingual arch. This article describes the design, mechanism of action and the relative advantages and disadvantages of the technique used.
\end{abstract}

Keywords: Buccally impacted premolar, Lingual arch, Asymmetric malalignment.

How to cite this article: Tahilramani SM, Gaikwad S, Karandikar G, Ravindranath VK. Lingual Approach to Buccally Impacted Teeth. J Contemp Dent 2014;4(3):162-166.

Source of support: Nil

Conflict of interest: None

\section{INTRODUCTION}

The word impaction is from Latin origin-Impactus. Impaction is defined as the cessation of eruption of a tooth caused by a physical barrier or ectopic positioning of a tooth. An impacted tooth is one that is erupted, partially erupted or unerupted and will not eventually assume a normal arch relationship with the other teeth and tissues. ${ }^{1}$

Teeth can be impacted due to local, genetic and systemic causes. ${ }^{2,3}$

Local causes for impaction of teeth include lack of space due to over retained deciduous tooth, lack or space for eruption in the arch, irregular position of tooth and/or adjacent teeth, ankylosis of teeth or even softtissue interferences. ${ }^{2}$

Impaction of teeth, although not a very rare occurrence, is still perhaps one of the more challenging tasks for an orthodontist to treat efficiently.

\footnotetext{
${ }^{1}$ Postgraduate Student, ${ }^{2}$ Senior Lecturer, ${ }^{3}$ Professor

${ }^{4}$ Professor and Head

${ }^{1-4}$ Department of Orthodontics, MGM Dental College and Hospital, Navi Mumbai, Maharashtra, India
}

Corresponding Author: Samay Mukesh Tahilramani, Postgraduate Student, Department of Orthodontics, MGM Dental College and Hospital, Navi Mumbai, Maharashtra, India, Phone: 9833101316, e-mail: samaynhdc@gmail.com
Teeth that are more commonly involved in impaction are the permanent maxillary and mandibular third molars, the permanent maxillary canines and less frequently the premolars or the incisors.

Treatment of teeth that are impacted presents a completely different challenge to the orthodontists in terms of diagnosis, treatment planning as well as the mechanics involved. ${ }^{4}$

\section{CASE REPORT}

\section{History}

A 14-year-old patient reported with a chief complaint of incomplete eruption of a lower left tooth in the posterior region. There was no significant medical or dental history reported and the patient reported in good physical and mental health.

\section{Diagnosis}

A 14-year-old male patient with class I skeletal bases, average growth pattern, class I molar and canine relationship on the left side, end on molar relationship on the right side, over retained 53, overjet of $4 \mathrm{~mm}$, overbite of $4 \mathrm{~mm}$, palatally impacted 13 and buccally impacted 35 (Fig. 1).

\section{Treatment Objectives}

The treatment objectives included alignment of the impacted mandibular premolar and the maxillary canine and achieve a well-balanced functional occlusion.

\section{Treatment Alternatives}

The space and the cephalometric analysis of the case warranted a nonextraction therapy for both the arches. The impacted premolar could be aligned in a traditional manner by aligning the remaining teeth with a rectangular stainless steel wire in place and then bringing the impacted tooth into alignment.

\section{Treatment Plan}

Nonextraction treatment was planned, involving extraction of only the over retained deciduous tooth after the levelling and aligning of the maxillary arch as that would act as a natural space maintainer for the eruption of the palatally impacted tooth. 

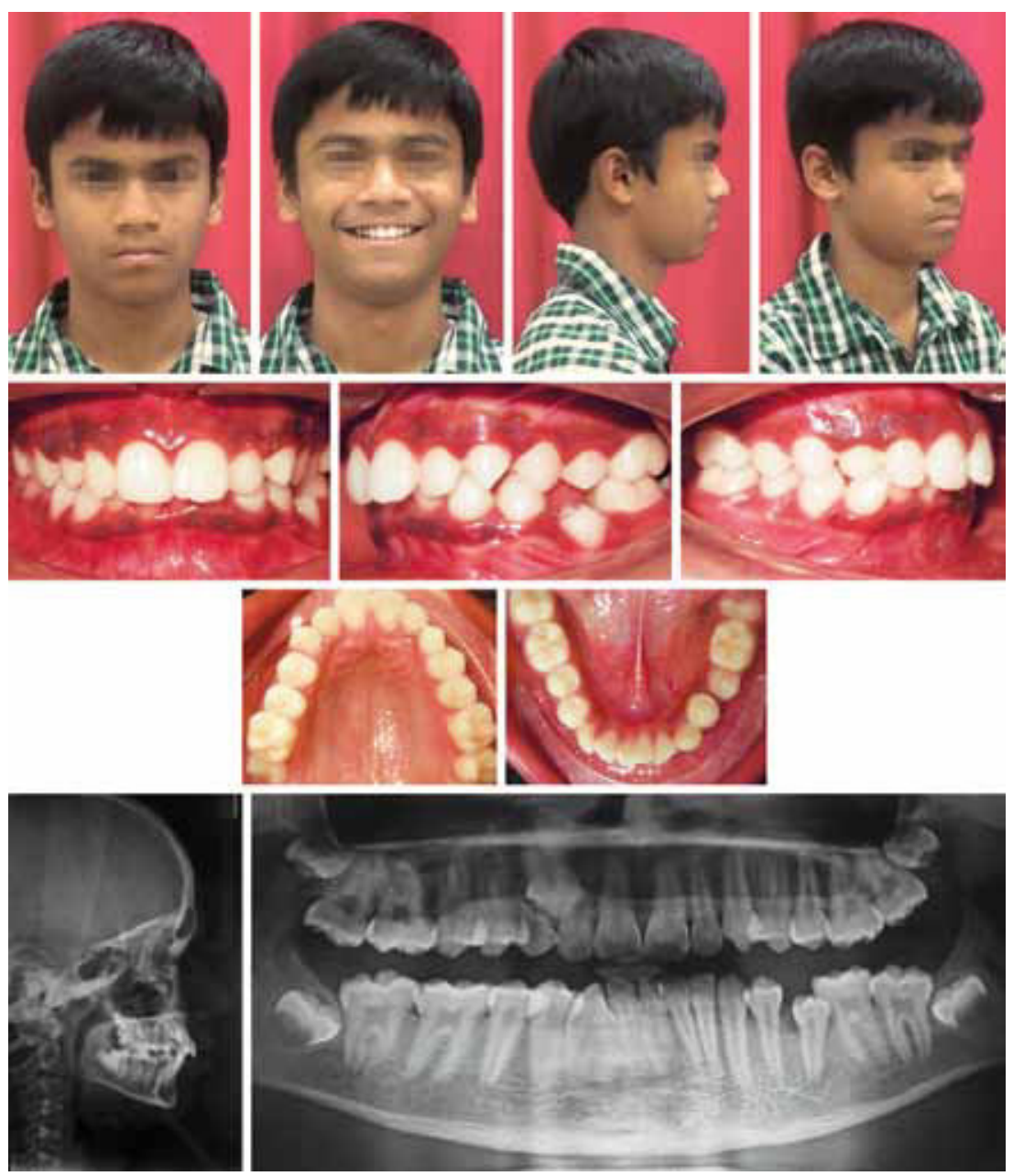

Fig. 1: Case MP: pretreatment photographs and radiographs

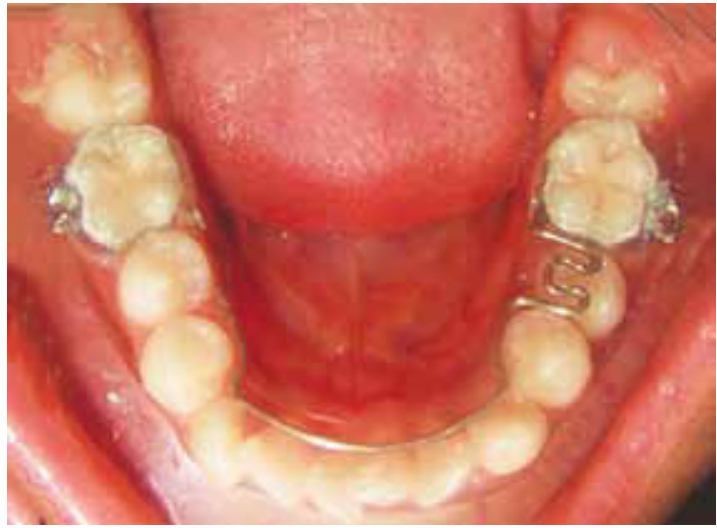

Fig. 2A: Modified lingual arch appliance design

\section{Treatment Progress}

Fixed mechanotherapy using pre-adjusted edgewise appliance (PEA) with a 0.022" slot was planned for the patient. However, for the mandibular arch, the treatment was initiated for the impacted premolar (35) at the beginning itself using the modified lingual arch (Figs $2 \mathrm{~A}$ and $\mathrm{B})$.

A Begg's bracket was bonded to the impacted premolar and a force element was provided using 0.010"

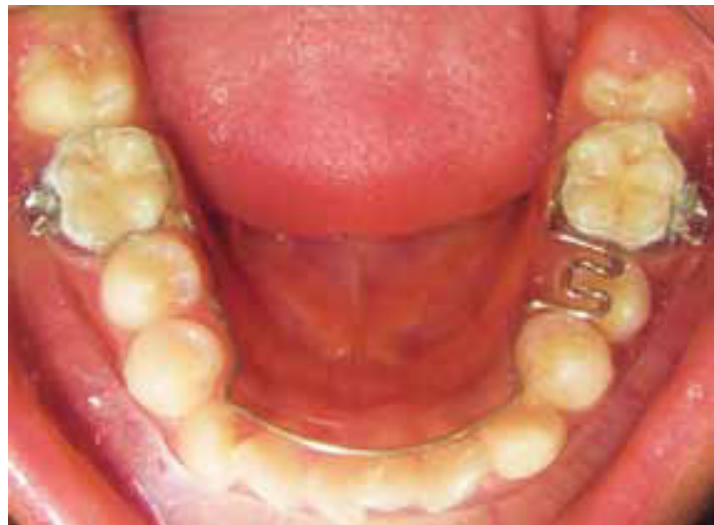

Fig. 2B: Modified lingual arch cemented intraorally

ligature that extended from the loops of the lingual arch to the vertical slot of the Begg's bracket (Fig. 3A). The buccal view of the premolar is seen after 10 days of application of the ligature (Fig. 3B).

Bonding of the maxillary and mandibular arches was done and 0.014" NiTi was placed. Note that the impacted premolar was not involved with the main arch wire (Figs $4 \mathrm{~A}$ and $\mathrm{B})$. The patient was recalled after a period of 3 weeks. 


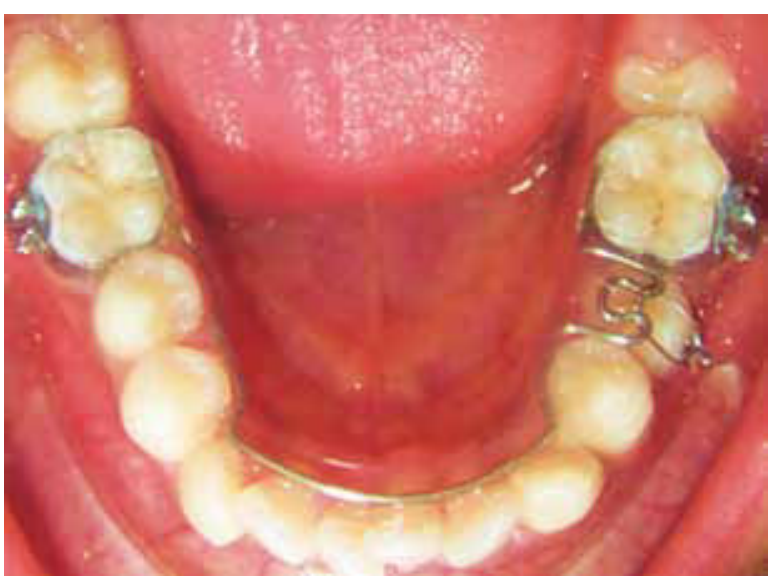

Fig. 3A: Begg's bracket bonded to 35 and traction using 0.010" ligature

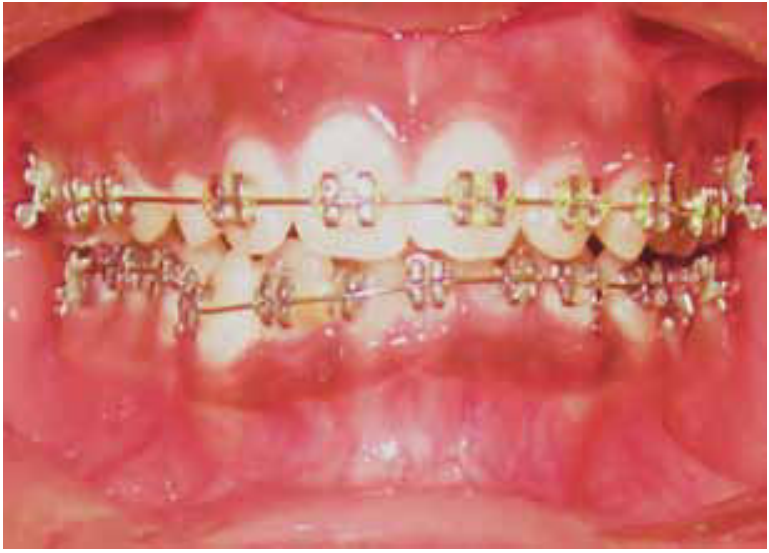

Fig. 4A: Bonding of maxillary and mandibular arches

At the recall visit 3 weeks later, the impacted premolar was seen to have erupted considerably as a result of the ligature traction (Fig. 5).

A 0.018 " NiTi was placed in the maxillary and $0.016^{\prime \prime}$ NiTi was placed in the mandibular arch. At this stage, the modified lingual arch was removed by separating the soldered joint between the molar bands and the wire component of the lingual arch using a carbide bur at slow speed with water coolant. The mandibular 0.016" $\mathrm{NiTi}$ wire was now passed beneath the vertical slot of the Begg's bracket (Figs 6A and B).

The patient was recalled after a period of 4 weeks. At this appointment, 0.018" AJ Wilcock was placed in both the arches (Fig. 7A) and the patient was appointed for a surgical exposure of the palatally impacted canine (13).

\section{TREATMENT RESULTS}

By about 8 weeks after the initiation of treatment, the impacted premolar was already aligned. While the impacted canine (13) is being scheduled an appointment for the surgical exposure, the impacted premolar (35) has already been aligned and is in occlusion with its counterpart in the maxillary arch (Fig. 7B).

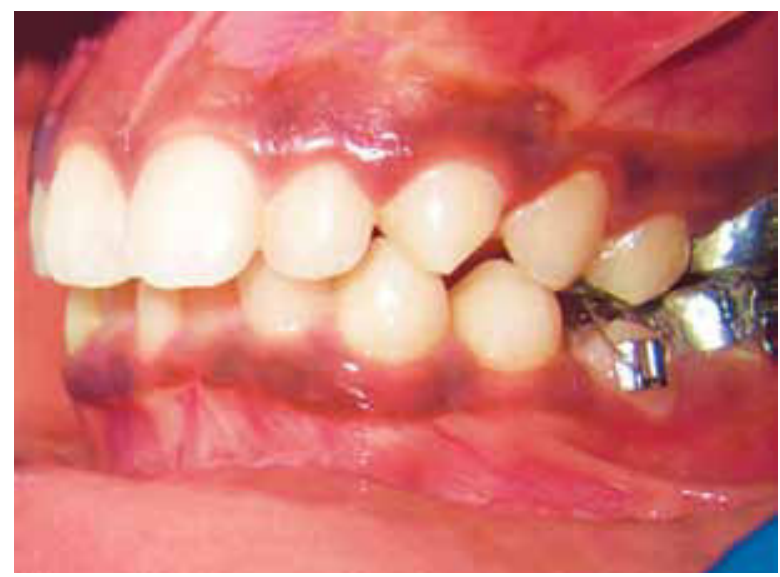

Fig. 3B: Buccal view of the premolar at the 10th day following ligature

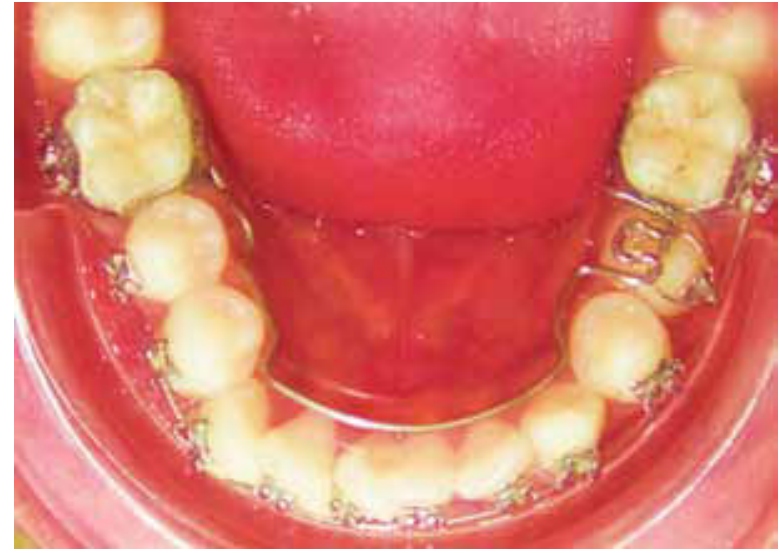

Fig. 4B: Thirty-five is not involved with the main arch wire

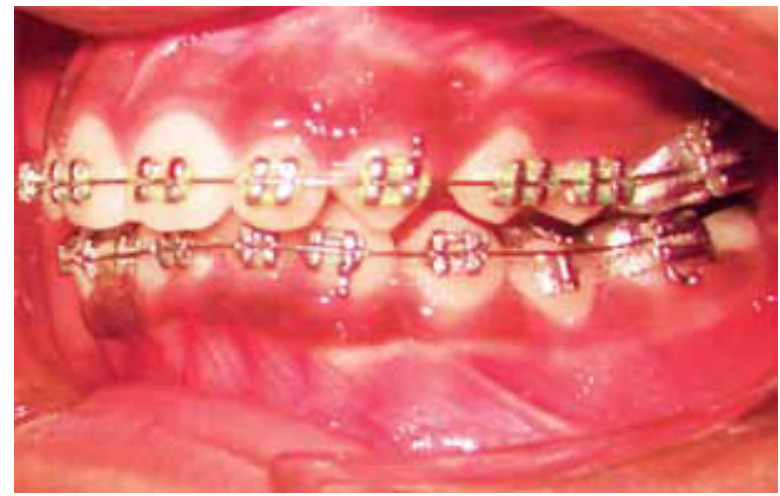

Fig. 5: Impacted 35 has erupted considerably

The palatally impacted canine was surgically exposed, a Begg's bracket was subsequently bonded and ligature was placed (Fig. 8).

\section{DISCUSSION}

An unerupted or an impacted tooth is an extreme example of an asymmetric malalignment problem, with one tooth far from the line of occlusion. ${ }^{6}$ If a superelastic NiTi wire is tied to the impacted tooth as well as the teeth adjacent to it, then the teeth adjacent to the impacted tooth will tip into the space where the impacted tooth is 


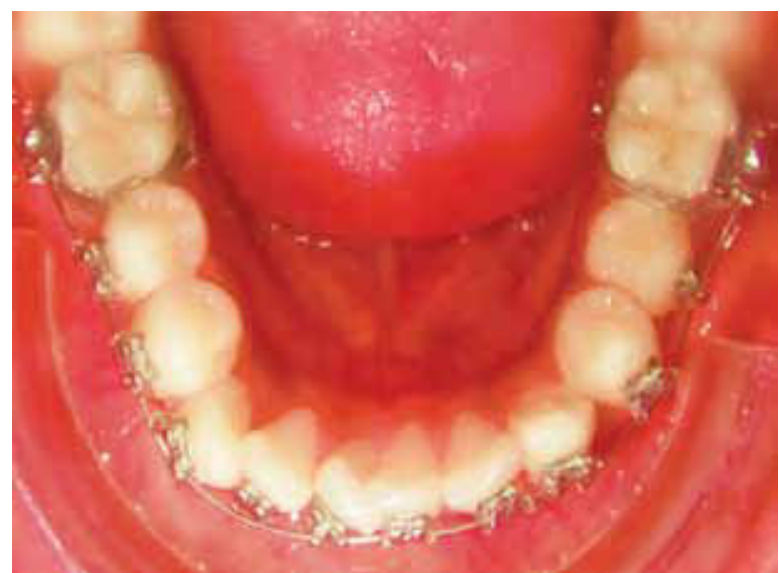

Fig. 6A: Modified lingual arch removed bracket

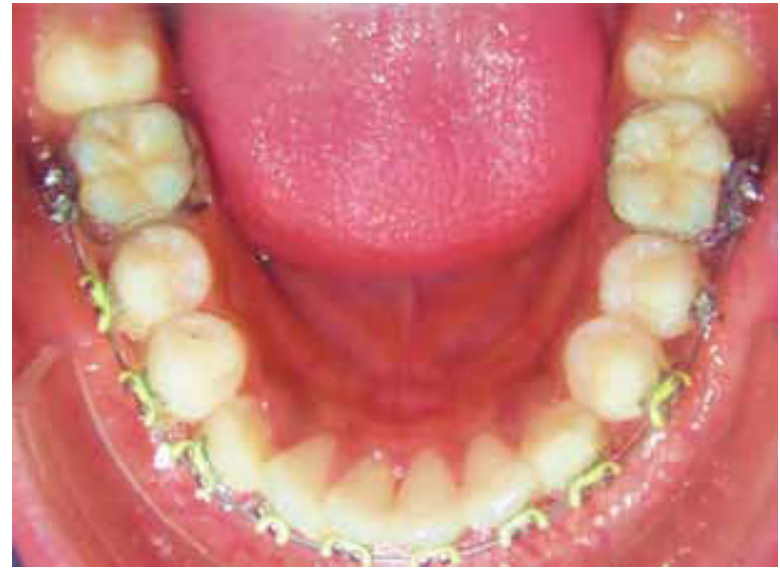

Fig. 7A: A 0.018" AJ Wilcock placed beneath Begg's bracket slot

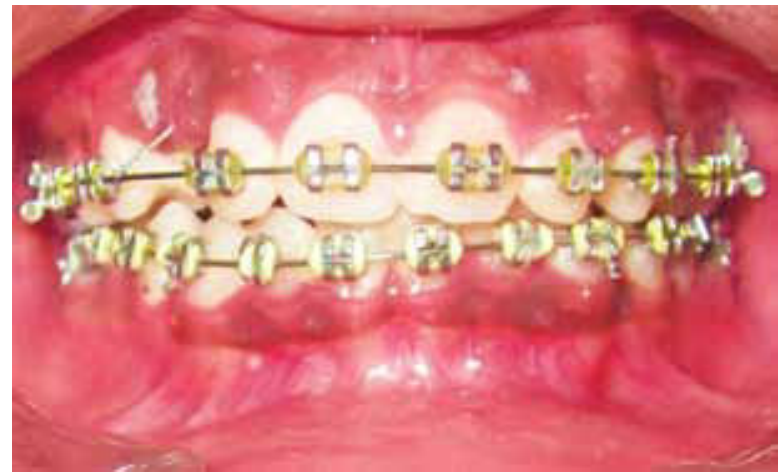

Fig. 8: Surgical exposure of maxillary canine. A 0.018" AJ Wilcock in both the arches

meant to come into alignment. Thus, a heavy stabilizing archwire must be present to allow the remaining teeth to act as the anchor unit, before the force is applied to the unerupted tooth. ${ }^{5,6}$ This would mean alignment of the crowded teeth for about 2 to 3 months when a rigid wire like 0.018 " stainless steel or a rectangular stainless steel wire can be engaged. Until then, the unerupted tooth cannot be given any force to aid eruption into the space.

The modified lingual arch precludes the need to level and align the remaining teeth, allowing the alignment

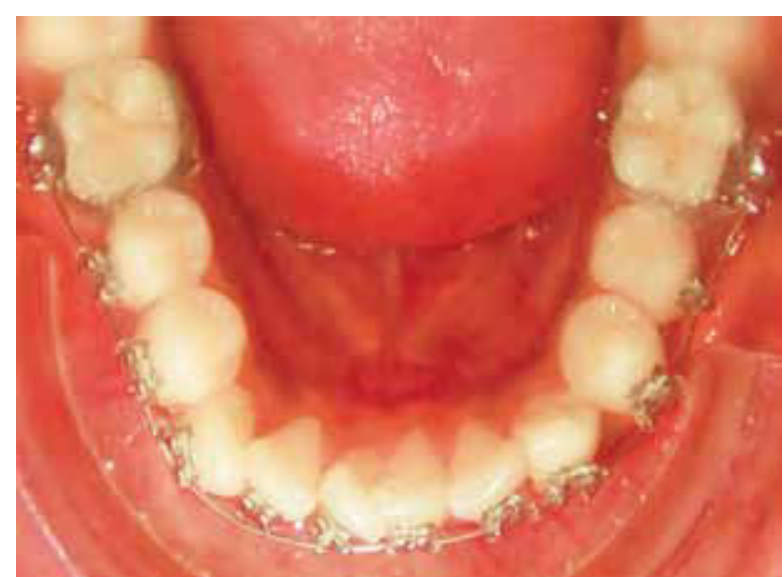

Fig. 6B: A 0.016" NiTi engaged into the slot of Begg's bracket

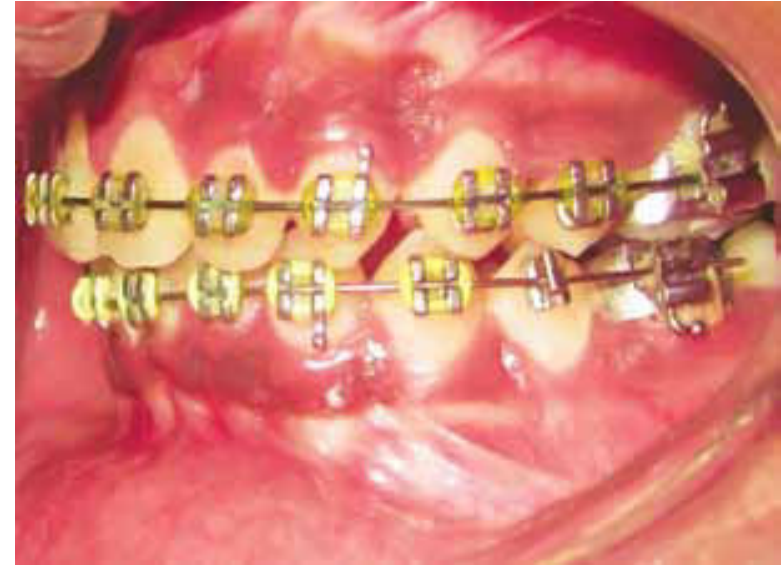

Fig. 7B: The premolar in occlusion with its maxillary counterpart

of the unerupted tooth to begin at the initiation of orthodontic treatment itself.

It is essentially an example of two different mechanics acting at the same time, both of them, being independent of the other. One part of the mechanics shown in the above case report is the traditional alignment process of the crowded teeth, with brackets bonded to all teeth, and wire sequences traditionally followed for alignment. The other part of the mechanics, used exclusively for aligning the unerupted premolar is carried out with the modified lingual arch.

The modified lingual arch is fabricated using $1 \mathrm{~mm}$ diameter stainless steel wire (Fig. 2A). Bands are fabricated on the mandibular first molars. Then, a mandibular impression is made to fabricate the modified lingual arch to be soldered from the first molar band on one side to the first molar band on the other side. The modified lingual arch is then cemented in place after fabrication (Fig. 2B). The design is 'modified' in the region of the unerupted premolar. Extensions of the lingual arch are made (Fig. 2A) that extend toward the buccal surface of the teeth. The extensions are fabricated such that they lie at the 
level of the occlusal plane, thus do not interfere with the occlusion as well as prevent the supra eruption of the opposing tooth. The above extensions, passively contact the proximal surfaces of the teeth adjacent to the premolar, thus acting as a space maintainer for the unerupted tooth as well.

With the lingual arch in place, the unerupted premolar can now be given a traction, without the risk or danger of the adjacent teeth tipping into the space or the opposing tooth supra erupting. Thus, the adjacent teeth are aligned using the traditional mechanics with the superelastic NiTi wires, and at the same time, the unerupted premolar is given traction achieving alignment of the same.

Eventually, with no tipping of adjacent teeth and no loss of space, the unerputed tooth has been completely aligned, allowing it to be engaged with the stainless steel wire as a part of the complete arch aligning mechanics.

The purpose of this article is to describe a different technique for eruption of the unerupted or impacted teeth using a modification of the lingual arch. It essentially describes the technique of having two different mechanics acting simultaneously and independent of each other, thus serves an essential tool to save treatment duration and avoid undesirable side effects. The complexity of the design could be a possible disadvantage to the operator and may contribute to patient discomfort, however we did not experience any such problems.

\section{CONCLUSION}

- The modified lingual arch, as suggested can be an effective appliance for the vertical eruption of unerpted teeth.

- The use of simultaneous, yet independent mechanics to align unerpted or impacted teeth is essential to achieve desired results in the shortest possible time without undesired tooth movement or loss of space.

- The technique described can be useful tool to save time as well as avoid undesirable side effects of aligning an unerpted or an impacted tooth.

\section{REFERENCES}

1. Malik NA. 2nd ed. Textbook of Oral and Maxillofacial Surgery. Jaypee Brothers Medical Publishers Pvt Ltd, 2012; 122-124.

2. Becker A. The Orthodontic Treatment of Impacted Teeth. 2nd ed. Martin Dunitz 2007:4-6.

3. Fournier A, Turcotte JY, Bernard C. Orthodontic considerations in the treatment of maxillary impacted canines. Am J Orthod 1982 March;81(3):236-239.

4. Bishara SE. Impacted maxillary canines: a review. Am J Orthod Dentofac Orthop 1992 Feb;101(2):159-171.

5. Sinha PK, Nanda RS. Management of impacted maxillary canines using mandibular anchorage. Am J Orthod Dentofac Orthop 1999 Mar;115(3):254-257.

6. Proffit W. 4th ed. Contemporary Orthodontics. St Louis: CV Mosby 2007;565-567. 